\title{
ITS and Trnl-F Sequences Analysis of Pulsatilla.Dahurica (Fisch.) Spr.
}

\author{
Tiantian WANG \\ Laboratory of Molecular Genetics \\ Institute of Frontier Medical Sciences \\ Jilin University \\ Changchun, China \\ e-mail:ttw14@mail.jlu.edu.cn \\ Shaoxuan ZHANG* \\ Laboratory of Molecular Genetics \\ Institute of Frontier Medical Sciences \\ Jilin University \\ Changchun, China \\ e-mail: zhangshaoxuan@jlu.edu.cn
}

\author{
Guanzhun LIN \\ Cardiolvascular Disease Diagnosis and Treatment Center \\ The First Bethune Hospital \\ Jilin University \\ Changchun, China \\ e-mail: lingz6403@163.com
}

\author{
Yarong LI \\ Department of Oncology and Hematology \\ The Second Bethune Hospital \\ Jilin University \\ Changchun, China \\ e-mail: doctor.lyr@163.com
}

\begin{abstract}
To provide scientific data of the internal transcribed spacer (ITS) and trnL-F sequences for the authentication of Pulsatilla chinesis (Bge.) Regel., we extracted the genome DNA from the leaves of Pulsatilla.dahurica (Fisch.) Spr., collected in Jilin Province, amplified the ITS and trnL-F region using ITS universal and trnL-F primers of angiosperm, and sequenced the purified PCR products directly. The obtained sequences were edited by Genetyx and reported here.
\end{abstract}

Keywords-Pulsatilla.dahurica (Fisch.) Spr.; ITS; trnL-F; Sequencing: Authentication.

\section{INTRODUCTION}

Chinese Anemone, the dried herb of Pulsatilla chinesis(Bge.) Regel.(Ranunculaceae), has the functions of detoxicating, removing heat from the blood and relieving diarrhea.It has been prescribed in every edition of «Chinese Pharmacopoeia»[1].Although only Pulsatilla chinesis(Bge.) egel is prescribed in "Chinese Pharmacopoeia", due to the historical reasons, there are still many area in China use different species of Pulsatilla such as Pulsatilla koreana Nakai., Pulsatilla.dahurica (Fisch.) Spr., (distributed in northeast of China); Pulsatilla turiczaninovii Krylov et Serg., Pulsatilla ambigua Turez ex Pritz (distributed in Inner Mongolia, Xinjiang of China) as Chinese Anemone [2].These lead to mistakes in collecting and application, and affect the therapeutic effect of Chinese Anemone. So authentication of Pulsatilla chinesis(Bge.) Regel from the others is necessary for correct use of this drug. Although methods based on traditional appearance, microscopy and thin-layer chromatography have been established and even improved, but due to the similar morphology and the same chemical composition, it is very hard to authenticate Chinese Anemone from the others used as it by them. Recently, as a result of progresses in molecular biology, molecular authentication has become a new and very reliable method. An accurate method of molecular authentication is generally established based on the data of DNA sequences, such as ITS and chloroplast genes like trnL-F, so sequencing of these regions of Pulsatilla chinesis(Bge.) Regel and the other plants that are used as Chineses Anemone becomes the most foundamental step. In this study, we sequenced the ITS and trnL-F region of Pulsatilla.dahurica (Fisch.) Spr. The obtained sequences were edited and reported here.

\section{MATERIALS AND METHODS}

\section{A. Materials}

Pulsatilla.dahurica (Fisch.) Spr. was collected in Jilin Province, China. The sample was authenticated by Professor Wenchang Guo of Jilin University (Table I). The leaves dried by silica gel were used for DNA extraction.

\section{B. DNA Extraction}

$0.3 \mathrm{~g}$ leaf was taken from every sample, and put into a culture dish. The leaves were cleaned with cotton swabs to wipe out the impurities with $70 \%$ of alcohol. The dried leaves were then grinded to powders in liquid nitrogen and used to extract genome DNA using Plant DNA Isolation Reagent (Takara Biotechnology (Dalian) Co., Ltd., Dalian, China) following the manufacturer's manual. The extracted genome DNA was dissolved in adequate volume of ultrapure water. The quality of the DNA was checked by $1 \%$ agarose gel electrophoresis with $5 \mu \mathrm{L}$ of each solution.

\section{Primer Design}

The universal primers of ITS [3] and trnL-F [4] were selected and synthesized by Takara Biotechnology (Dalian)

Co., Ltd., (Dalian China). The sequences are as follows:

PCR primers:

For ITS:

ITS-F: 5'-TCC ACT GAA CCT TAT CAT TTA G-3'

ITS-R: 5'-CCA TGC TTA AAC TCA GCG GGT-3' 
For trnL-F:

trnLF-cF: 5'-CGAAATCGGTAGACGCTACG-3'

trnLK-fR: 5'-ATI'TGAACTGGTGACACGAG-3'

Sequencing primers:

For ITS: ITS-F: 5'-TCC ACT GAA CCT TAT CAT TTA G-3' In-ITS-3'R:5'-GAC TCG ATG GTT CAC GGG ATT CT-3' In-ITS-5'F: 5'-TCT CGC ATC GAT GAA GAA CG-3' ITS-R: 5'-CCA TGC TTA AAC TCA GCG GGT-3'

For trnL-F: trnLF-cF: 5'-CGAAATCGGTAGACGCTACG-3' trnLF-dR: 5'-GGGGATAGAGGGACTTGAAC-3' trnLK-eF: 5'-GGTTCAAGTCCCTCTATCCC-3' trnLK-fR: 5'-ATI'TGAACTGGTGACACGAG-3'

\section{PCR Amplification}

PCR was performed in a $50 \mu \mathrm{L}$ reaction mixture containing $1 \mu \mathrm{L}$ of each C's PCR primer, $5 \mu \mathrm{L}$ of template DNA, $5 \mu \mathrm{L}$ of $10 \times$ Buffer, $5 \mu \mathrm{L}$ of dNTPs, $1 \mu \mathrm{L}$ of Taq DNA polymerase (Takara Biotechnology (Dalian) Co., Ltd., Dalian China). PCR was performed in the following conditions: 1 cylce of $94^{\circ} \mathrm{C}$ for $5 \mathrm{~min}, 35$ cycles of denature at $94^{\circ} \mathrm{C}$ for $1 \mathrm{~min}$, annealing at $55^{\circ} \mathrm{C}$ for $2 \mathrm{~min}$, extension at $72^{\circ} \mathrm{C}$ for $2 \mathrm{~min}, 1$ cycle of $72^{\circ} \mathrm{C}$ for $10 \mathrm{~min}$. All the reactions were performed using MiniCycler PTC-150(MJ Research Inc, St. Bruno, Canada). All the PCR products were checked using a $1 \%$ agarose gel.

\section{E. Sequencing}

D's PCR products were purified using Montage PCR Filter Units (Millipore Corporation, USA.) and then sequenced. Sequencing reaction was performed in a $10 \mu \mathrm{L}$ reaction mixture by using ABI BigDye Terminator v3.1 Cycle Sequencing Kit (Applied Biosystems, USA.) with each of the sequencing primers. Sequencing reaction was performed in the following conditions: 1 cycle of $96^{\circ} \mathrm{C}$ for 1 min, 25 cycles of denature at $96^{\circ} \mathrm{C}$ for $30 \mathrm{sec}$, extension $50^{\circ} \mathrm{C}$ for 5 sec. 1 cycle of $60^{\circ} \mathrm{C}$ for $4 \mathrm{~min}$. After purification, sequences were analyzed by using 3130 sequencer (Applied Biosystems, USA.).

\section{F. Sequence Analysis}

Genetyx-SV/RC version 11.0 (Software Development Co., Ltd., Tokyo, Japan) were used for editing of the sequences.

\section{RESULTS}

A. The Information of The Purchased Plants Is Shown in Table I. Three Individuals of Each Sample Were Used for Sequence Analysis.
TABLE I. THE LOCATION AND DATE OF SAMPLE COLLECTION

\begin{tabular}{ccc}
\hline Species & Location & Date of collection \\
\hline $\begin{array}{c}\text { Pulsatilla. } \\
\text { dahurica (Fisch.) } \\
\text { Spr. }\end{array}$ & Changqing Road & \\
\hline
\end{tabular}

B. The ITS Sequences of Pulsatilla Dahurica (Fisch.) Spr.Are as Follows:

TCGATGCCTGCTCAGCAGAACGACCCGCGAACAAG TGAAAACACCAACTCACGCCGGGGAACAGGACGC CAGACAGCCTCACCGCTGCCCCCGACCTGCGCCCC AGCACAACAAAAAAAATCCGGCGCAACTGGCGCC AAGGAATACTTACCGGAAACAACGGGTTGACACGT CGACGCCGTGGATCCGAATACTCAAACGACTCTCG GCAACGGATATCTCGGCTCTTGCATCGATGAAGAA CGTAGCGAAATGCGATACTTGGTGTGAATTGCAGA ATCCCGTGAACCATCGAGTCTTTGAACGCAAGTTG CGCCCGAAACCTTTCTGGTCGAGGGCACGTCTGCC TGGGCGTCACACACAGCGTCGCCCCCACCAAAGCA TTTGGATGGGGGCGGAAATTGGCCCCCCGAGCCCC CCGGGGCACGGTCGGCACAAATGTTGGCCCTCGGC GGCGAGCGTCGCGGTTAGCGGTGGTTGTACTCTCA TCCTCCAAAGACAAAATGACGCGTCCGCCTCGTCG CCCGCTGGGCRAAGATGACCCAAGGAGTCTCCCCT ACCGGTCTTCCACCT

$\mathrm{R}: \mathrm{A} / \mathrm{G}$

C. The Trnl-F Sequences of Pulsatilla.Dahurica (Fisch.) Spr.Are as Follows:

TCCTGAGCMAATCCTTATTTCAGAAAACAAAAGAG GGTTCAGAAAGCAAGAATAAAAAAAGGATAGGTG CAGAGACTCAATGGAAGCTGTTCTAACGAATAGAG TTGACTGCGTTGATCAAGGATTCCTTTTAAACTACA GAAAGGCTGAACCCGTATACATATAATACAAAAAT ATCAAAGATTAAAAGATTAATGGTCATCCAAATCT TTATTTTTTCTTATATGATATACAAAATGGAAGAAT TCTTGTGATGTGAATCGATTCCAAGTTTAAGTAAG AATCGAATATTCACTGATCAAATAAGTCACTCTAT AATCTGATAGTTCTTTTAAAGAACTAATTGGACGA GAATAAAGATAGAGTCCCATTATACATGTCAATCC CAATACTGACAAAAATGAAATTTATAGTAAGAGGA AAATCCGTCGACTTTTGAAATCGTGAGGGTTCAAG TCССTCTATCCСCATCCCCAATAAAAAGTTTGCTGT ACCCCCTAACTATTTAGTTTTAGTTAATGTTAATTA ATTAAAAGTCATTTTTTTTTTTTTTTAGATTAGTTAT GTTTCTCAGCCATTCTACTCTTTCACAAACGGATCG TGGGAGAAAAGGCCTCTCTTATCACAAGACTTGTG ATATATTGTGATATATACAATATATATGTGATATGT GTAAATCAACATCTAGGAGAAAGGAATTCCCATTT GAATCATTCACGTTTAATATAATTATTTAAACTTAC AAACAAAGTATTCTTTTTGTTTGAAGATCCAAGAC CAAGAAATTCCAGGGTTTGGGTAAGACTTTGTAAT GCTTTTTAGTCTATTTAATTGATATACCCAACAAGT ACTCTAAACGAGTACTCTAAATAGAGTGGGGATGC CGCATCGGGAAGAGTCGGGATAGCTCAGTGGTAGA GCAGAGGACGAA. 


\section{CONCLUSION}

1. The ITS and trnL-F sequences of Pulsatilla.dahurica (Fisch.) Spr.collected in China is for the first time reported.

2. This study provided scientific data for molecular authentication of Pulsatilla chinesis(Bge.) Regel.

3. Pulsatilla chinesis(Bge.) Regel. distributed in wide area of China, there should be differences among the samples collected in different area, so this study is just a beginning. For authenticating Pulsatilla chinesis(Bge.) Regel. more precisely, enlargement of sample from various area and accumulation of these data should be necessary in the future.

\section{REFERENCES}

[1] China Pharmacopoeia Commisson, Chinese Pharmacpoeia. Beijing, China: China Medical Science Press, 2010

[2] New Medical College of Jiangsu, A Dictionary of Traditional Chinese Medicine, Shanghai, China:Shanghai Sience and Technology Press,1985.

[3] F. Takaiwa, K. Oono, M. Sugiura,"Nucleotide sequence of the 17S25S spacer region from rice rDNA”, Plant Molecular Biology.Vol. 4 , 1985, pp.355-364, doi: 10.1007/BF02418257

[4] P. Taberlet, L. Gielly, G. Pautou, J. Bouvet, "Universal primers for amplification of three non-coding regions of chloroplast DNA" Plant Molecular Biology, Vol. 17, 1991, pp.1105-1109. 